\title{
Care-seeking and delay of care during COPD exacerbations
}

\author{
Emily R. Locke $\mathbb{1}^{1 凶}{ }^{1}$, Jessica P. Young ${ }^{1}$, Catherine Battaglia ${ }^{2,3}$, Tracy L. Simpson ${ }^{4,5}$, Ranak Trivedi ${ }^{6,7}$, Carol Simons ${ }^{1}$, John C. Fortney ${ }^{1,5}$,
} Paul Hebert ${ }^{1,8}$, Erik R. Swenson ${ }^{9,10}$, Jeffrey Edelman ${ }^{9,10}$ and Vincent S. Fan ${ }^{1,10}$

Patients who receive earlier treatment for acute exacerbations of chronic obstructive pulmonary disease (COPD) have a better prognosis, including earlier symptom resolution and reduced risk of future emergency-department visits (ED) or hospitalizations. However, many patients delay seeking care or do not report worsening symptoms to their healthcare provider. In this study, we aimed to understand how patients perceived their breathing symptoms and identify factors that led to seeking or delaying care for an acute exacerbation of COPD. We conducted semistructured interviews with 60 individuals following a recent COPD exacerbation. Participants were identified from a larger study of outpatients with COPD by purposive sampling by exacerbation type: 15 untreated, 15 treated with prednisone and/or antibiotics in the outpatient setting, 16 treated in an urgent care or ED setting, and 14 hospitalized. Data were analyzed using inductive content analysis. Participants were primarily male (97\%) with a mean age of $69.1 \pm 6.9$ years, mean FEV $1.42( \pm 0.63)$, and mean mMRC dyspnea of $2.7( \pm 1.1)$. We identified 4 primary themes: (i) access and attitudinal barriers contribute to reluctance to seek care, (ii) waiting is a typical response to new exacerbations, (iii) transitioning from waiting to care-seeking: the tipping point, and (iv) learning from and avoiding worse outcomes. Interventions to encourage earlier care-seeking for COPD exacerbations should consider individuals' existing self-management approaches, address attitudinal barriers to seeking care, and consider health-system changes to increase access to non-emergent outpatient treatment for exacerbations.

\section{Clinical Trial Registration NCT02725294}

npj Primary Care Respiratory Medicine (2022)32:7 ; https://doi.org/10.1038/s41533-022-00269-9

\section{INTRODUCTION}

Persons with chronic obstructive pulmonary disease (COPD) frequently experience acute exacerbations that are associated with decreased quality of life $^{1}$ and can lead to emergencydepartment (ED) visits and hospitalizations ${ }^{2}$. The economic impact of severe exacerbations is significant. In the United States in 2010 there were an estimated 1.5 million ED visits and 699,000 COPD hospitalizations with a mean cost of $\$ 8,228$ per hospitalization ${ }^{3,4}$.

Patients with exacerbations may require pharmacologic treatment, including bronchodilators, corticosteroids or antibiotics ${ }^{5}$. Earlier treatment can lead to more rapid symptom recovery and fewer ED visits and hospitalizations ${ }^{1,6}$. However, in one study, more than $70 \%$ of patients presenting to the ED with a COPD exacerbation waited more than $24 \mathrm{~h}$ before seeking care, thereby increasing the risk of hospitalization ${ }^{6}$. Moreover, an estimated $40 \%$ of patients do not report all their exacerbations to their healthcare providers, also increasing the risk of COPD hospitalization ${ }^{1}$.

COPD self-management programs that include an action plan with prescriptions for home antibiotics and prednisone for treatment of exacerbations have been shown to improve quality of life and reduce hospitalizations ${ }^{7,8}$. In a randomized controlled trial of a COPD exercise program where all participants had a COPD action plan, those initiating prednisone and antibiotics within 3 days of new respiratory symptoms had more rapid resolution of symptoms, but no difference in healthcare utilization than those who delayed treatment ${ }^{9}$. However, this study found that even with home prescriptions, $60 \%$ delayed treatment ${ }^{9}$. In a different study, patients with an action plan delayed treatment by an average of 6-7 days ${ }^{10}$. Other approaches to identifying COPD exacerbations such as telemonitoring programs have not consistently reduced ED visits or hospitalizations for COPD ${ }^{11}$. Therefore, a better understanding of why some patients delay treatment may help to improve COPD selfmanagement programs and to help develop more effective telemonitoring programs.

The reasons for not reporting worsening symptoms to the healthcare team or delaying care may be that patients do not recognize symptoms of exacerbations ${ }^{12}$. Additionally, the desire to avoid hospitalizations may be a key factor in choosing not to seek care $^{13,14}$. Qualitative studies of patients in the United Kingdom, Denmark, and the Netherlands found that barriers to seeking care included reluctance to see the doctor, not wanting to bother the doctor, lack of continuity of care, not knowing when it is appropriate to call, and difficulty traveling to the clinic ${ }^{12,15,16}$.

Given the potential benefits of early treatment during a COPD exacerbation, we utilized a qualitative descriptive design to understand how persons with COPD in a US health care system emotionally and cognitively perceived and managed their breathing symptoms, and the barriers and facilitators that led to seeking or delaying care for an acute exacerbation of COPD.

\footnotetext{
${ }^{1}$ Center of Innovation for Veteran-Centered and Value-Driven Care VA Puget Sound Health Care System, Seattle, WA, United States. ${ }^{2}$ Eastern Colorado VA Health Care System, Aurora, CO, United States. ${ }^{3}$ University of Colorado Anschutz Medical Campus, Aurora, CO, United States. ${ }^{4}$ Center of Excellence in Substance Addiction Treatment and Education (CESATE), VA Puget Sound Health Care System, Seattle, WA, United States. ${ }^{5}$ Department of Psychiatry, University of Washington School of Medicine, Seattle, WA, United States. ${ }^{6}$ Center for Innovation to Implementation, VA Palo Alto Health Care System, Palo Alto, CA, United States. ${ }^{7}$ Department of Psychiatry and Behavioral Sciences, Stanford University, Stanford, CA, United States. ${ }^{8}$ Department of Health Systems and Population Health, University of Washington, Seattle, WA, United States. ${ }^{9}$ Pulmonary and Critical Care,

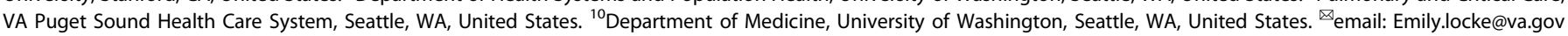




\section{METHODS}

Participants in this qualitative study were identified from a prospective observational study of 410 outpatients with COPD enrolled in primary care at two Veterans Affairs (VA) Medical Centers. Participants had COPD confirmed by spirometry $\left(\mathrm{FEV}_{1} /\right.$ FVC $<0.70), \geq 1$ treated COPD exacerbation in the previous year, and were English-speaking. Nursing-home residents and those with a diagnosis of dementia or Alzheimer's disease were excluded. Institutional review board approval was obtained at VA Puget Sound and Eastern Colorado VA and all participants provided written informed consent; standard ethical and researchgovernance procedures were followed. We adhered to the consolidated criteria for reporting qualitative research (COREQ) guidelines (Supplement 1) ${ }^{17}$.

Participants were contacted every 2 weeks to screen for worsening COPD symptoms. Exacerbations were defined as new or worsening breathing symptoms that lasted more than $48 \mathrm{~h}^{18,19}$. Among the participants who experienced exacerbations during the study, we identified individuals eligible to participate in this qualitative study if they had exacerbations that met the following criteria: (i) untreated (did not seek care or take corticosteroids or antibiotics) exacerbations with worsening symptoms that persisted for $\geq 7$ days and $<6$ weeks and were rated as moderately to extremely serious using the Response to Symptoms Questionnaire ${ }^{20}$ modified for COPD; (ii) treated with oral corticosteroids and/or antibiotics in the outpatient setting; (iii) treated in an urgent care or ED setting; or (iv) hospitalized. During January 2017 through February 2018, we purposively sampled from all 4 exacerbation categories soon after the exacerbation ended. Individuals were invited to participate in a phone-based, semistructured qualitative interview. Recruitment was discontinued when saturation occurred, and no new concepts or themes related to the study aims were identified ${ }^{21}$.

\section{Data collection}

Interviews conducted by trained qualitative researchers (JY and CS) were digitally recorded and transcribed (interview length 32-65 min, average $48 \mathrm{~min}$ ). The interview guide started with broad, open-ended questions, followed by more specific questions and probes to elicit thorough, detailed descriptions of exacerbation episodes. The questions examined perceived need for care for a COPD exacerbation based on Leventhal's Common Sense Model to understand how individuals responded both cognitively and emotionally to worsening breathing symptoms ${ }^{22}$. Participants were asked to describe the course of their recent exacerbation including symptoms, their emotional and cognitive responses, coping strategies, symptom management, care-seeking, treatment received, involvement of others (such as caregivers, family, and friends), and comparison to past exacerbations and care-seeking behaviors (see Supplement 2 for Interview Guide). Interviewers also created written field notes for each interview to capture contextual information, encourage interviewer reflection, and facilitate interviewer technique improvement ${ }^{23}$.

\section{Analysis}

Data were analyzed using inductive content analysis, an approach in which themes are derived from the data through open coding, code grouping, categorization, and identifying patterns of meaning across the data ${ }^{24,25}$. Experienced qualitative analysts (CS and JY) closely reviewed all transcripts and created an initial code list based on meaning units identified in the data. The coding framework was refined through discussion between the analysts and larger research team and coding discrepancies were resolved through consensus building. All data were coded using Atlas.ti qualitative software (version 8) and sorted into categories based on similarities and differences within and across interviews and themes were identified based on patterns in the data.

Interview data were iteratively revisited to refine themes, ensure findings were grounded in the data, and validate the results ${ }^{26}$. The entire study team, which included clinicians from a variety of disciplines and health-service researchers with expertise in COPD, reviewed analytic results at multiple time points to finalize themes, confirm the validity and credibility of findings, and identify representative quotes.

\section{Reporting summary}

Further information on research design is available in the Nature Research Reporting Summary linked to this article.

\section{RESULTS}

Sixty participants were interviewed: (i) 15 untreated, (ii) 15 treated in the outpatient setting, (iii) 16 treated in the ED or urgent care clinic, and (iv) 14 hospitalized. The study cohort was mainly male $(97 \%)$ with a mean age of $69.1 \pm 6.9$ years, mean $\mathrm{FEV}_{1} 1.42( \pm 0.63)$ liters, and mean mMRC dyspnea of $2.7( \pm 1.1)$ (Table 1).

Four primary themes and 7 subthemes related to care seeking and delay of care were identified. The results are presented by theme and include select representative quotations (Table 2).

\section{Theme 1: Attitudinal and access barriers contributed to reluctance to seek care}

When recounting exacerbation episodes, participants consistently reported a reluctance to seek care and the desire to "put off" careseeking. This reluctance was based on a combination of attitudinal and access-related barriers to care.

Attitudinal barriers to care. Participants described avoiding careseeking because of an attitude of self-reliance and not wanting to "bother" or "burden" others. Wanting to "go it alone" and concerns about the impact of exacerbations on family and clinicians were commonly described as contributing to hesitancy to seek care $(Q 1, Q 2)$.

Practical barriers to accessing in-person care. The "hassle" or "inconvenience" of seeking care was commonly cited as a motivation for delaying care-seeking and comprised a combination of practical issues such as distance to care, concerns with driving during an exacerbation, time associated with seeking and receiving care, and missing work or neglecting other responsibilities. Although each of these factors alone was not a significant barrier to care, participants described their additive nature as contributing to a pervasive reluctance toward careseeking (Q3, Q4).

Heightened reluctance to seek care in ED settings, but few other options. Many participants described reluctance to seek care in emergency settings due to amplified practical concerns (such as greater travel distance and time spent waiting for treatment) and a belief that the ED is appropriate only in "true emergencies" like severe shortness of breath (Q5). For some, this reluctance impacted care-seeking and translated into increased delays in care (Q6). Although individuals frequently reported wanting to receive treatment in outpatient settings, many said they encountered wait times for outpatient appointments that precluded being seen in primary care during an acute COPD exacerbation (Q7). Additionally, many participants reported calling for a primary care appointment only to be instructed to seek care in the ED instead. Some participants explained that a history of being referred from primary to emergency care made it less likely that they would contact primary care for future exacerbations (Q8). 
Table 1. Demographics of interviewed participants.

\begin{tabular}{|c|c|c|}
\hline & \multicolumn{2}{|l|}{$N=60$} \\
\hline & $\mathrm{N}$ or mean & $\%$ or SD \\
\hline \multicolumn{3}{|l|}{ Demographics } \\
\hline Age & 69.1 & \pm 6.9 \\
\hline Male gender & 58 & 96.7 \\
\hline Race, Caucasian & 47 & 78.3 \\
\hline Income $>\$ 20,000$ per year & 40 & 70.2 \\
\hline Currently employed & 10 & 16.7 \\
\hline Education, college graduate & 19 & 31.7 \\
\hline Live with others & 43 & 71.7 \\
\hline Living as a couple & 40 & 66.7 \\
\hline Dual Medicare and VA user & 47 & 78.3 \\
\hline \multicolumn{3}{|l|}{ COPD severity } \\
\hline $\mathrm{FEV}_{1}$ (liters) & 1.42 & \pm 0.63 \\
\hline $\mathrm{FEV}_{1} \%$ predicted & 43.6 & \pm 19.4 \\
\hline \multicolumn{3}{|l|}{$\mathrm{FEV}_{1} \%$ predicted by severity category } \\
\hline$\geq 80 \%$ (Mild) & 4 & 6.7 \\
\hline 50-79\% (Moderate) & 16 & 26.7 \\
\hline 30-49\% (Severe) & 25 & 41.7 \\
\hline 0-29\% (Very severe) & 15 & 25.0 \\
\hline mMRC dyspnea scale & 2.7 & \pm 1.1 \\
\hline \multicolumn{3}{|l|}{$\begin{array}{l}\text { Type of exacerbation treatment during the one-year } \\
\text { follow-up period }\end{array}$} \\
\hline$\geq 1$ Untreated & 47 & 78.3 \\
\hline$\geq 1$ treated as outpatient & 30 & 50.0 \\
\hline$\geq 1$ urgent care or ED & 23 & 38.3 \\
\hline$\geq 1$ Hospital & 22 & 36.7 \\
\hline \multicolumn{3}{|l|}{ COPD treatment } \\
\hline Chronic corticosteroid use, oral & 7 & 11.7 \\
\hline Home oxygen therapy & 31 & 51.7 \\
\hline Home nebulizer use & 31 & 52.5 \\
\hline Pulmonary provider ${ }^{a}$ & 38 & 63.3 \\
\hline $\begin{array}{l}\text { At-home prescription for corticosteroids and/or } \\
\text { antibiotics }\end{array}$ & 14 & 23.3 \\
\hline $\begin{array}{l}\text { Prior participation in a pulmonary rehabilitation } \\
\text { program }\end{array}$ & 16 & 26.7 \\
\hline $\begin{array}{l}\text { Enrolled in the VA care coordination home } \\
\text { telehealth program for COPD }\end{array}$ & 3 & 5.0 \\
\hline \multicolumn{3}{|l|}{ Access to emergency care } \\
\hline Minutes to VA emergency department & 62.7 & 38.7 \\
\hline Minutes to non-VA emergency department & 15.9 & 9.4 \\
\hline
\end{tabular}

Theme 2: Waiting is a typical response to new exacerbations

Participants described being aware when a worsening breathing episode began and recognized early exacerbation symptoms such as fatigue, shortness of breath, increased difficulty in breathing, chest tightness, increased sputum production, and/or coughing. Many participants described having their own typical symptompresentation patterns, and attributed "knowing when something [exacerbation] is coming on" to their history of living with COPD and their experience with recurrent exacerbations (Q9). Almost all participants described waiting as a normative, typical response to the onset of a new exacerbation and new symptoms, with the following subthemes:
"Trying everything first". All participants described addressing new exacerbation symptoms with home-based treatments or symptom management prior to care-seeking. Patients often described developing their home-treatment approach through experimentation and experience; few reported having been provided with or following specific clinical instruction, although a minority of patients had been prescribed at-home medications (prednisone or antibiotics) to take for exacerbations. This "first line of defense" included using over-the-counter and on-hand prescribed medications, increasing home oxygen levels, increasing inhaler use, and/or adding "stronger" respiratory treatments like nebulizers and resting (Q10) (Q11). Participants described implementing these treatments singly or in combination, and waiting to see what, if any, effect they had on symptoms. For some, a variety of management approaches were tried, or layered together, over time (Q12). The individuals who were prescribed prednisone or antibiotics to have on-hand often reported trying other selfmanagement approaches before "resorting" to taking them during an exacerbation due to concerns about overreliance on antibiotics or steroids and perceived negative side effects (Q13).

"Waiting it Out". Participants also commonly described waiting to see if their symptoms would subside in the hope of avoiding care-seeking. Some explained that the courses of their exacerbations were unpredictable and varied, with some resolving without incident and others worsening and requiring medical attention (Q14). However, many participants acknowledged that hopes of avoiding care-seeking and the desire to "wait it out" often led them to overestimate the chances that episodes would improve and to ignore or downplay worsening symptoms (Q15).

Waiting but not waiting too long. Many participants acknowledged potential negative outcomes related to delayed careseeking and described a balance between wanting to wait for care but not wanting to "wait too long." "Waiting too long" was defined as allowing episodes to progress to the point of negative outcomes such as urgent care-seeking (e.g., panicking, activating emergency medical services [calling 911], and having to be rushed to the ED), advanced illness (e.g., pneumonia, passing out from breathlessness), and/or hospitalization (Q16). Many described a desire to wait until the symptoms were "bad enough" to necessitate medical treatment but not serious enough to end in crisis. However, waiting too long was common and many described episodes in which waiting resulted in negative outcomes (Q17).

\section{Theme 3: Transitioning from waiting to care-seeking: the tipping point}

All participants who sought treatment described a tipping point at which they transitioned from waiting and managing exacerbation symptoms to active care-seeking. Participants who did not seek care reported that their symptoms improved and never reached a tipping point, although some acknowledged that an episode could have easily gotten worse and considered themselves "lucky" that they improved (Q18). Each person who sought care had their own criteria for care-seeking, and participants often reported applying their individual tipping point consistently across exacerbations (Q19). Individuals initiated care-seeking in the following four common circumstances:

Self-management strategies were exhausted. Some participants reported seeking care when symptoms worsened or failed to improve, despite best efforts at home-based treatment of their exacerbation episode. Individuals often described seeking care only when they had used all their available inhalers and medications and had "nothing left to try" (Q20). 
Table 2. Themes and corresponding exemplar quotes.

Theme 1: Access and attitudinal barriers contribute to reluctance to seek care Attitudinal barriers to care

Q1 I don't like asking anybody for anything...they'd take me I know, but I just hate asking them. I don't involve Pt 37 anybody in it. There's family here, but I don't involve them. It's my problem.

Q2 I don't want to trouble the paramedics...or the hospital...every time I have these things, there's a feeling of Pt 45 guilt...putting people through this ...going to the doctor, going to the hospital, having to call 911, putting my wife through it.

\section{Practical barriers to accessing in-person care}

Q3 It's just a hassle - driving through traffic, the time it takes, paying for gas, missing work... I don't go unless I Pt 7 absolutely have to.

Q4 Yeah, just the inconvenience of going there...it's not that I can't go, there's no one thing stopping me... I Pt 11 just don't want to do it...it's such a bother.

\section{Heightened reluctance to seek care in ED settings, but few other options}

Q5 The ER, that's the place to go only if you are in real dire straits, you don't go there if your breathing is just Pt 55 bad, only if you absolutely can't get a breath...so you put it off if you can.

Q6 I don't like going to the doctor but I hate going to the ER...I try to keep out of there as long as I possibly can. Pt 46

Q7 First I called them up [Primary Care office] but they said it would be a couple weeks before I could see Pt 2 anyone...couldn't wait that long with my breathing.

Q8 Sure, if I could call up and see someone [in primary care], I would do that but that's not how it works...you Pt 20 can't get in to see someone when you need it. They just say to go to the ED. ... I don't even call them anymore.

\section{Theme 2: Waiting is a typical response to new exacerbations}

Q9 I've been dealing with this so long...l always know when it's getting started and something is coming on.... Pt 49 always starts the same way...get tight in the chest, hard to take a deep breath.

\section{"Trying everything first"}

Q10 I can tell when something is coming on so I do everything...my inhalers, my oxygen, my medications and I Pt 38 try and slow down and rest. Everything I can do myself first [before seeking care].

Q11 So I know what to do...I take my two inhalers, and if it still continues then I go up to the store and pick up Pt 42 some Claritin-D, some Sudafed, some Musinex...then after a while my breathing will open up and after a few days of those I'm a lot better. [LATER] Oh I've just figured out what works through trial and error...I've been dealing this this [COPD] long enough that I've just figured out what works for me.

Q12 I have some medications my doctor gave me to use here when I need them [steroids and antibiotics]. I Pt 23 started on those after my breathing got bad and gunky... and I did all my other usual stuff like my inhalers. Then you wait and see what happens... You gotta give that stuff time to try and work it out.

Q13 My doctor has given me things I can take if I need them [antibiotics] but I don't like resorting to using them Pt 33 ...don't think it's good to use them too much... don't like how they make me feel and I don't think it's good for you.

\section{"Waiting it Out"}

Q14 I just do all my usual stuff and wait for it to pass... sometimes it gets better and sometimes it don't. 3

Q15 Because you always seem to hope that it's just a cough or cold, and then it just keeps getting bad and then Pt 59 you have to go to the doctor. You want to think it will get better, but usually it doesn't.

\section{Waiting but not waiting too long}

Q16 What happens is if I wait too long, if I fight it and try to do it with my own medications...then I have to go to Pt 14 the hospital... then I think I've waited too long. When I had to call the ambulance, I should've gone earlier.

Q17 In my case, a lot of times l'd wait a week or two weeks before l'd go in. A lot of time l'd wait to the point Pt 18 where it'll take forever to fix. If I hadn't waited, if I'd gotten there sooner, it would've been fixed sooner and it wouldn't have gotten so bad.

\section{Theme 3: Transitioning from waiting to care-seeking: The tipping point}

Q18 This time it didn't get so bad. At about day seven, just when I was thinking I might need to head to the ED, I Pt 53 started breathing a little better. ... Wasn't nothing I did, just got lucky this time I guess.

Q19 I always wait a week, that's the time I give it each time my breathing gets bad. Then if it's not better at a Pt 40 week I call the doctor.

Q20 If what I'm doing just is not working and I'm using my inhalers here like crazy... and if it's not working and I Pt 1 have nothing left to try, then I better get going.

Q21 Yeah, when I couldn't catch my breath even when I laid still... when I couldn't even walk across the room or Pt 10 get to the bathroom, I decided this is something I couldn't handle by myself. So I went to the ER.

Q22 I can usually tell by what I'm coughing up, like a rainbow. And if it gets to be a certain color, I call my Pt 28 doctors.

Q23 I turned my machine all the way up and still couldn't breathe...my rescue inhaler didn't work. I was gasping Pt 19 for air and got all panicky which made it worse, so I ended up taking an ambulance to the hospital.

Outpatient treatment

Untreated

Hospital

Outpatient treatment

ED

Outpatient treatment

Hospital

Hospital

Untreated

Untreated

ED

ED

Outpatient treatment

Hospital 


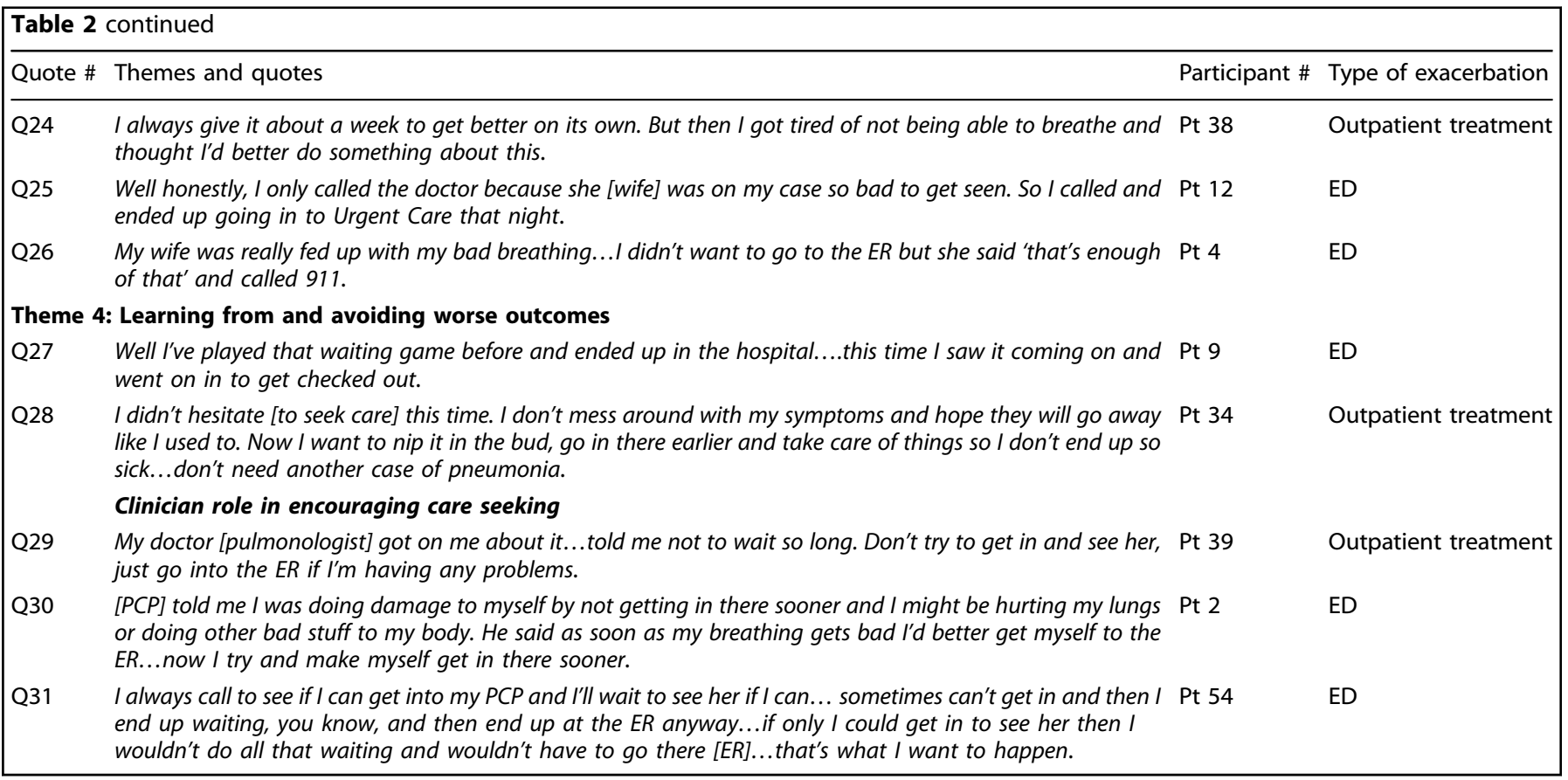

Severe or worrisome symptoms. Participants commonly described waiting until their symptoms worsened and became urgent, unbearable, or "bad enough" to necessitate care-seeking. Extreme breathlessness (e.g., gasping for breath, unable to walk across the room) (Q21), and unusual, observable, "worrisome signs" (e.g., sputum color, temperature, and coughing leading to bleeding or vomiting) (Q22), were commonly described as triggering careseeking. Breathlessness was sometimes accompanied by panic or anxiety, which often resulted in urgent care-seeking, such as calling 911 (Q23).

Episodes went on longer than anticipated. Participants often described a typical time they waited before seeking care, and episodes that exceeded this duration prompted care-seeking. While most reported they would wait approximately a week before seeking care, the duration of symptoms prior to careseeking ranged widely from 3 days to $2 \frac{1}{2}$ weeks (Q24).

A family member encouraged or insisted upon care-seeking. Some participants described seeking care only with the encouragement or insistence of family members (e.g., wives or adult children). Often, participants did not believe their symptoms warranted medical treatment and acknowledged that they sought care only to appease or address the concerns of others (Q25). Although family-member involvement typically prompted participant care-seeking behaviors, a few participants described episodes in which family members sought care (such as calling a doctor or 911) without the participant's instruction (Q26).

\section{Theme 4: Learning from and avoiding worse outcomes}

Although many participants described consistently delaying careseeking during COPD exacerbations, some reported that their care-seeking patterns evolved over time. The desire to avoid repeating past experiences with worse outcomes like hospitalization and calling 911 sometimes served as a powerful motivator toward earlier care-seeking (Q27). Some explained that they learned to be more proactive in their care-seeking in order to "nip it [exacerbation] in the bud" and avoid more severe illness like pneumonia (Q28).

Clinician role in encouraging care-seeking. A few participants reported that clinicians played an important role in underscoring the impact of worse outcomes and encouraging earlier careseeking. Clinical encounters following a negative outcome like hospitalization or calling 911 affected some individuals' understanding of when, where, and why to seek earlier care during an exacerbation (Q29, Q30). Some participants stated that having access to a primary care provider visit within a few days of calling, as opposed to needing to go to the ED, would facilitate earlier treatment (Q31).

\section{DISCUSSION}

In this study, a reluctance to seek care was a typical or normative response of many persons with COPD when they developed symptoms of an exacerbation. Participants generally sought care when self-management strategies were exhausted, severe symptoms developed, episode duration was longer than expected, and/or family members encouraged care-seeking. Participants did not seek care until they reached a tipping point when their perceived need for care overcame the attitudinal and access barriers they faced.

Similar to our results, a reluctance to seek care has been documented in several qualitative studies of individuals with COPD in which themes of stoicism and wishing to not bother their providers contributes to delays in seeking care ${ }^{12,15,16,27}$. Our study also suggests that treatment setting can impact an individual's reluctance or willingness to seek care. Many participants described a heightened reluctance to seek care in the ED for practical and attitudinal reasons and preferred to be seen in a primary care setting during an exacerbation. However, participants often were unable to make timely primary care appointments and were told to seek care in the ED, which they commonly sought to avoid.

Prior studies found that distinguishing between COPD exacerbations and daily fluctuation of baseline symptoms may be challenging, especially for older adults ${ }^{28}$. Qualitative studies of 
individuals with heart failure ${ }^{29}$ and $\mathrm{COPD}^{30}$ found that individuals who had difficulty recognizing symptoms and/or attributing them to an exacerbation often delayed seeking care. However, a study of COPD patients found that patients were generally able to identify exacerbations based on worsening symptoms ${ }^{31}$. Similarly, in our study, participants did not have difficulty recognizing exacerbation symptoms, and were able to readily distinguish the typical symptom onset from their baseline breathing. All participants in the study cohort experienced at least one treated exacerbation in the year prior to the study, which may have made it more likely to recognize new exacerbations.

Upon recognizing the symptoms, participants described a typical approach of waiting to see whether exacerbation symptoms would respond to at-home treatments that were often based on trial-and-error approaches through experience with prior exacerbations rather than clinical instruction. Participants in our study were not enrolled in a formal COPD self-management program, although $27 \%$ had previously participated in a pulmonary rehabilitation program, $5 \%$ were enrolled in a VA telemonitoring program, and $23 \%$ reported receiving a home prescription for prednisone or antibiotics. Waiting, often for one or more weeks, until their symptoms either improved or became unbearable or severe was part of their self-management strategy, and at times led to a crisis requiring individuals to be hospitalized. Williams and colleagues reported that individuals' prior experience managing exacerbations played an important role in determining how they identified and managed exacerbations. Similar to our study, participants preferred to manage their symptoms by themselves at home and felt confident monitoring their symptoms based on their experiential knowledge of living with COPD for many years ${ }^{30,31}$.

Laue and colleagues reported that COPD exacerbation selftreatment was a means of avoiding seeking medical care. When at-home treatment failed, some patients further delayed seeking care as they were worried it would be viewed as a personal failure $^{32}$. A study by Korpershoek and colleagues found that some patients recognized exacerbation symptoms early and were likely to initiate prompt self-management and follow-up with a healthcare provider, whereas others lacked self-management strategies and were less likely to seek care ${ }^{30}$. The factors associated with care-seeking included self-empowerment and trusting their primary care provider whereas those who delayed care reported being stubborn or not wanting to bother their provider, as well as ambivalence toward treatment due to negative side effects of prednisone ${ }^{30}$.

Although earlier treatment of COPD exacerbations can lead to more rapid resolution of worsened breathing symptoms and may reduce the likelihood of ED visits and hospitalizations ${ }^{1,9}$, even with self-management training, some patients still delay care $^{9,10}$. Therefore, it is important to incorporate individuals' experiences with prior exacerbations to reduce barriers to seeking care sooner. Our study suggests that strategies to address attitudinal barriers such as self-reliance and wanting to avoid being a burden to others may be needed. For example, promising results from an open trial of motivational interviewing (MI)-based health coaching ${ }^{33}$ and a small trial of $\mathrm{MI}$ to improve treatment adherence suggest that MI may help address these attitudinal barriers ${ }^{34}$.

In addition to addressing patient characteristics, our findings suggest that healthcare systems need to address the "hassle" aspects of seeking care and other perceived logistical barriers to accessing care to make it easier for individuals to choose to seek care earlier. Such system-level improvements might include increasing options for evaluation and treatment in outpatient clinical settings, including options for virtual care, to provide alternatives to going to the ED. Additionally, follow-up visits after hospitalizations and ED visits may provide opportunities to help individuals with COPD improve self-management skills and careseeking behaviors.

Another approach to encourage early care-seeking is to incorporate family caregivers who are important in COPD management and treatment planning ${ }^{35,36}$. Providing education on when and where to seek treatment, and support for family members in their caregiving roles may be beneficial ${ }^{37}$. Given that most participants in our study were male veterans, and many family caregivers are likely female, care-seeking strategies may need to take these gender dynamics into account. However, further exploration of the efficacy and benefits of this strategy is needed as caregivers may lack agency, knowledge, and support to best help individuals with COPD, and attempts to influence earlier care-seeking could cause relational tension or conflict ${ }^{38}$.

It is possible that underlying attitudes around care-seeking during an exacerbation may differ among people who regularly seek care compared with those who opt to "wait-it-out" without taking corticosteroids or antibiotics or seeing a provider. A key strength of this study is that we interviewed participants who received different types of care for their exacerbations, including those who successfully recovered without formal treatment, those treated as outpatients, and those with more severe exacerbations treated in the ED or as inpatients. We noted themes shared among the four treatment groups around the desire to wait and attempt to manage symptoms at home. Participants who had not sought care for an exacerbation stated that they had not reached the tipping point at which they would seek care.

A limitation of this study was that most participants were male veterans, and these findings may not generalize to the greater population of individuals with COPD. Older male veterans represent a cultural group that includes traits associated with masculinity and military culture such as stoicism, concealing vulnerability and non-help-seeking behaviors ${ }^{39,40}$. While similar traits of stoicism and self-reliance have been noted among nonveterans with $\operatorname{COPD}^{27,41}$, differences in care-seeking attitudes may exist. The risk of exacerbations and care-seeking behaviors of female patients with COPD may be different as well ${ }^{42,43}$. Indeed, one study found that women with COPD were more likely to delay presenting to the ED compared with men ${ }^{44}$, while another study of COPD hospitalizations at VA facilities found that compared with men, women were less likely to receive some inhaled medications, with shorter hospital stays and lower readmission rates ${ }^{45}$. It remains to be seen, however, whether the diverse population of people who live with COPD would report the same kinds of attitudes about care-seeking as we found in our US sample of predominantly male veterans. As COPD is a global public health challenge, further research is needed to understand how contextual factors such as different social conditions or cultures may influence seeking or delaying care and will be vital in shaping intervention strategies that are applicable to the diverse groups of people with COPD.

In summary, we found that reluctance to seeking care is a common response to exacerbations. Self-management approaches based on prior exacerbation experiences were often used before seeking care, and failure of these strategies was pivotal in the decision of when and where to seek care. Attitudinal barriers such as self-reliance along with perceived barriers to access to care, including the desire to avoid the emergency room, were important factors in delaying care. Interventions that seek to understand patients' lived experience with exacerbations, their attitudes toward seeking care, and address potentially suboptimal self-management approaches combined with healthcare-system changes to improve access to nonemergent outpatient treatment, may help to facilitate earlier intervention for COPD exacerbations. 


\section{DATA AVAILABILITY}

Due to ethical restrictions, we are unable to share data publicly because the data contain potentially identifying and/or sensitive patient information.

Received: 6 May 2021; Accepted: 22 December 2021;

Published online: 15 February 2022

\section{REFERENCES}

1. Wilkinson, T. M., Donaldson, G. C., Hurst, J. R., Seemungal, T. A. \& Wedzicha, J. A. Early therapy improves outcomes of exacerbations of chronic obstructive pulmonary disease. Am. J. Respir. Crit. Care Med. 169, 1298-1303 (2004).

2. Halpin, D. M., Miravitlles, M., Metzdorf, N. \& Celli, B. Impact and prevention of severe exacerbations of COPD: a review of the evidence. Int J. Chron. Obstruct Pulmon Dis. 12, 2891-2908 (2017).

3. Perera, P. N., Armstrong, E. P., Sherrill, D. L. \& Skrepnek, G. H. Acute exacerbations of COPD in the United States: inpatient burden and predictors of costs and mortality. COPD 9, 131-141 (2012).

4. Ford, E. S. et al. COPD surveillance-United States, 1999-2011. Chest 144, 284-305 (2013)

5. Goldcopd. Global Strategy for the Diagnosis, Management and Prevention of COPD 2020 Report (2020) http://goldcopd.org/.

6. Chandra, D., Tsai, C. L. \& Camargo, C. A. Jr. Acute exacerbations of COPD: delay in presentation and the risk of hospitalization. COPD 6, 95-103 (2009).

7. Bourbeau, J., Nault, D. \& Dang-Tan, T. Self-management and behaviour modification in COPD. Patient Educ. Couns. 52, 271-277 (2004).

8. Lenferink, A. et al. Self-management interventions including action plans for exacerbations versus usual care in patients with chronic obstructive pulmonary disease. Cochrane Database Syst. Rev. 8, CD011682 (2017).

9. Bischoff, E. W. et al. Effects of written action plan adherence on COPD exacerbation recovery. Thorax 66, 26-31 (2011).

10. Fan, V. S. et al. A comprehensive care management program to prevent chronic obstructive pulmonary disease hospitalizations: a randomized, controlled trial. Ann. Intern. Med. 156, 673-683 (2012).

11. Barbosa, M. T., Sousa, C. S., Morais-Almeida, M., Simoes, M. J. \& Mendes, P. Telemedicine in COPD: An Overview by Topics. COPD 17, 601-617 (2020).

12. Adams, R., Chavannes, N., Jones, K., Ostergaard, M. S. \& Price, D. Exacerbations of chronic obstructive pulmonary disease-a patients' perspective. Prim. Care Respir. J. 15, 102-109 (2006).

13. Robinson, K., Lucas, E., van den Dolder, P. \& Halcomb, E. Living with chronic obstructive pulmonary disease: the stories of frequent attenders to the emergency department. J. Clin. Nurs. 27, 48-56 (2018).

14. Lowey, S. E., Norton, S. A., Quinn, J. R. \& Quill, T. E. A place to get worse: perspectives on avoiding hospitalization from patients with end-stage cardiopulmonary disease. J. Hosp. Palliat. Nurs. 16, 338-345 (2014).

15. Gruffydd-Jones, K., Langley-Johnson, C., Dyer, C., Badlan, K. \& Ward, S. What are the needs of patients following discharge from hospital after an acute exacerbation of chronic obstructive pulmonary disease (COPD)? Prim. Care Respir. J. 16, 363-368 (2007).

16. Shipman, C., White, S., Gysels, M. \& White, P. Access to care in advanced COPD: factors that influence contact with general practice services. Prim. Care Respir. J. 18, 273-278 (2009)

17. Tong, A., Sainsbury, P. \& Craig, J. Consolidated criteria for reporting qualitative research (COREQ): a 32-item checklist for interviews and focus groups. Int. J. Qual. Health Care 19, 349-357 (2007).

18. Seemungal, T. A., Donaldson, G. C., Bhowmik, A., Jeffries, D. J. \& Wedzicha, J. A. Time course and recovery of exacerbations in patients with chronic obstructive pulmonary disease. Am. J. Respir. Crit. Care Med. 161, 1608-1613 (2000).

19. Leidy, N. K., Murray, L. T., Jones, P. \& Sethi, S. Performance of the EXAcerbations of chronic pulmonary disease tool patient-reported outcome measure in three clinical trials of chronic obstructive pulmonary disease. Ann. Am. Thorac. Soc. 11, 316-325 (2014)

20. Dracup, K. \& Moser, D. K. Beyond sociodemographics: factors influencing the decision to seek treatment for symptoms of acute myocardial infarction. Heart Lung 26, 253-262 (1997).

21. Sandelowski, M. Sample size in qualitative research. Res Nurs. Health 18, 179-183 (1995).

22. Diefenbach, M. A. \& Leventhal, H. The Common-sense model of illness representation: theoretical and practical considerations. J Soc. Distress Homeless 5, 11-38 (1996).

23. Phillippi, J. \& Lauderdale, J. A guide to field notes for qualitative research: context and conversation. Qual. Health Res. 28, 381-388 (2018).

24. Elo, S. \& Kyngas, H. The qualitative content analysis process. J. Adv. Nurs. 62 , 107-115 (2008)
25. Graneheim, U. H. \& Lundman, B. Qualitative content analysis in nursing research concepts, procedures and measures to achieve trustworthiness. Nurse Educ. Today 24, 105-112 (2004).

26. Morse, J. M., Barrett, M. A., Mayan, M., Olson, K. \& Spiers, J. Verification strategies for establishing reliability and validity in qualitative research. Int. J. Qual. Methods 1, 13-22 (2002).

27. Harrison, S. L. et al. "We are not worthy"-understanding why patients decline pulmonary rehabilitation following an acute exacerbation of COPD. Disabil. Rehabil. 37, 750-756 (2015).

28. Brandt, C. L. Study of older adults' use of self-regulation for COPD selfmanagement informs an evidence-based patient teaching plan. Rehabil. Nurs. 38 11-23 (2013).

29. Patel, H., Shafazand, M., Schaufelberger, M. \& Ekman, I. Reasons for seeking acute care in chronic heart failure. Eur. J. Heart Fail 9, 702-708 (2007).

30. Korpershoek, Y., Slot, J., Effing, T., Schuurmans, M. \& Trappenburg, J. Selfmanagement behaviors to reduce exacerbation impact in COPD patients: a Delphi study. Int. J. COPD In press (2017).

31. Williams, V., Hardinge, M., Ryan, S. \& Farmer, A. Patients' experience of identifying and managing exacerbations in COPD: a qualitative study. NPJ Prim. Care Respir. Med. 24, 14062 (2014).

32. Laue, J., Melbye, H. \& Risor, M. B. Self-treatment of acute exacerbations of chronic obstructive pulmonary disease requires more than symptom recognition - a qualitative study of COPD patients' perspectives on self-treatment. BMC Fam. Pract. 18, 8 (2017).

33. Rehman, H., Karpman, C., Vickers Douglas, K. \& Benzo, R. P. Effect of a motivational interviewing-based health coaching on quality of life in subjects with COPD. Respir. Care 62, 1043-1048 (2017).

34. Naderloo, H., Vafadar, Z., Eslaminejad, A. \& Ebadi, A. Effects of motivational interviewing on treatment adherence among patients with chronic obstructive pulmonary disease: a randomized controlled clinical trial. Tanaffos 17, 241-249 (2018).

35. Figueiredo, D., Gabriel, R., Jacome, C. \& Marques, A. Caring for people with early and advanced chronic obstructive pulmonary disease: how do family carers cope? J. Clin. Nurs. 23, 211-220 (2014).

36. Aasbo, G., Rugkasa, J., Solbraekke, K. N. \& Werner, A. Negotiating the care-giving role: family members' experience during critical exacerbation of COPD in Norway. Health Soc. Care Commun. 25, 612-620 (2017)

37. Caress, A. L., Luker, K. A., Chalmers, K. I. \& Salmon, M. P. A review of the information and support needs of family carers of patients with chronic obstructive pulmonary disease. J. Clin. Nurs. 18, 479-491 (2009).

38. Suresh, M., et al. Caregiver experiences and roles in care seeking during COPD exacerbations: a qualitative study. Ann. Behav. Med. 10.1093/abm/kaab045 (2021)

39. Plys, E., Smith, R. \& Jacobs, M. L. Masculinity and military culture in VA hospice and palliative care: a narrative review with clinical recommendations. J. Palliat. Care 35, 120-126 (2020).

40. Neilson, E. C., Singh, R. S., Harper, K. L. \& Teng, E. J. Traditional masculinity ideology, posttramatic stress disorder (PTSD) symptom severity, and treatment in service members and veterans: a systematic review. Psychol. Men. Masc. 21, 578-592 (2020).

41. Jones, I. et al. The needs of patients dying of chronic obstructive pulmonary disease in the community. Fam. Pract. 21, 310-313 (2004).

42. Stolz, D. et al. Differences in COPD exacerbation risk between women and men: analysis from the UK clinical practice research datalink data. Chest 156, 674-684 (2019).

43. Jenkins, C. R. et al. Improving the management of COPD in women. Chest 151, 686-696 (2017).

44. Cydulka, R. K. et al. Gender differences in emergency department patients with chronic obstructive pulmonary disease exacerbation. Acad. Emerg. Med. 12, 1173-1179 (2005).

45. Bade, B. C. et al. Sex differences in veterans admitted to the hospital for chronic obstructive pulmonary disease exacerbation. Ann. Am. Thorac. Soc. 16, 707-714 (2019).

\section{ACKNOWLEDGEMENTS}

This work was supported by IIR 14-060 from the United States (U.S.) Department of Veterans Affairs, Health Services Research and Development Service. The views expressed in this article are those of the authors and do not necessarily reflect the position or policy of the Department of Veterans Affairs or the United States Government. 


\section{AUTHOR CONTRIBUTIONS}

V.S.F., C.B., T.L.S., R.T., J.C.F., P.H, E.R.S., and J.E. were involved in the concept, design, and revising the paper. J.P.Y., C.S., E.R.L., and V.S.F. were involved in the data acquisition, analysis, interpretation, and drafting and revising the paper.

\section{COMPETING INTERESTS}

Dr. Fan received research funding from the Department of Veterans Affairs. The other co-authors have no competing financial interests to report.

\section{ADDITIONAL INFORMATION}

Supplementary information The online version contains supplementary material available at https://doi.org/10.1038/s41533-022-00269-9.

Correspondence and requests for materials should be addressed to Emily R. Locke.

Reprints and permission information is available at http://www.nature.com/reprints
Publisher's note Springer Nature remains neutral with regard to jurisdictional claims in published maps and institutional affiliations.

cc) (i) Open Access This article is licensed under a Creative Commons Attribution 4.0 International License, which permits use, sharing, adaptation, distribution and reproduction in any medium or format, as long as you give appropriate credit to the original author(s) and the source, provide a link to the Creative Commons license, and indicate if changes were made. The images or other third party material in this article are included in the article's Creative Commons license, unless indicated otherwise in a credit line to the material. If material is not included in the article's Creative Commons license and your intended use is not permitted by statutory regulation or exceeds the permitted use, you will need to obtain permission directly from the copyright holder. To view a copy of this license, visit http://creativecommons. org/licenses/by/4.0/.

This is a U.S. government work and not under copyright protection in the U.S.; foreign copyright protection may apply 2022, corrected publication 2022 